\title{
Astrocyte elevated gene-1 overexpression in human primary gallbladder carcinomas: An unfavorable and independent prognostic factor
}

\author{
WEI SUN ${ }^{1}$, YUE-ZU FAN ${ }^{1}$, HAO XI $^{2}$, XING-SUI LU $^{1}$, CHUN YE $^{1}$ and JING-TAO ZHANG ${ }^{1}$ \\ ${ }^{1}$ Department of Surgery, Tongji Hospital, Tongji University School of Medicine; ${ }^{2}$ Department of \\ Pathology, Shanghai Tenth People's Hospital, Tongji University, Shanghai, P.R. China
}

Received April 27, 2011; Accepted June 15, 2011

DOI: $10.3892 /$ or.2011.1387

\begin{abstract}
Astrocyte elevated gene-1 (AEG-1), as an HIV-1 or TNF- $\alpha$-inducible transcript, is correlated with various aspects of tumor malignancy. However, the status of AEG-1 expression and its clinical significance in human gallbladder carcinoma (GBC) remains unknown. In the present study, we investigated AEG-1 expression in two GBC cell lines (GBC-SD and SGC-996) and GBC tissues by immunohistochemical, Western blot and real-time PCR analysis. We found that AEG-1 was highly expressed in GBC samples (63.4\%, 26 of 41) compared with normal gallbladder mucosa $(\mathrm{p}=0.0003)$ and highly invasive GBC-SD cell lines at both the protein $(\mathrm{p}=0.0043)$ and mRNA levels $(\mathrm{p}=0.0001)$, and strongly correlated with differentiation degree $(\mathrm{p}=0.006)$, Nevin stage $(\mathrm{p}=0.0344), \mathrm{Ki}-67$ expression $(\mathrm{p}=0.0024)$ and liver infiltration $(\mathrm{p}=0.0332)$ in these patients. Multivariate analysis indicated that AEG-1 overexpression was an independent prognostic marker for GBC patients. Moreover, patients with high AEG-1 levels had shorter survival time $(\mathrm{p}=0.008)$. Our results suggest that the AEG-1 protein is a valuable marker of GBC progression and could be a potential therapeutic target.
\end{abstract}

\section{Introduction}

Gallbladder carcinoma (GBC) is the most common malignancy of the biliary tract, the fifth or sixth most common malignant neoplasm of the digestive tract and the leading cause of cancerrelated deaths in western countries, China and in Shanghai $(1,2)$. The effective therapy to cure gallbladder carcinoma is complete surgical resection at the early stage. However, only $10 \%$ of patients present with early-stage disease and are considered surgical candidates. In this context, the survival rate is as high

Correspondence to: Professor Yue-Zu Fan, Department of Surgery, Tongji Hospital, Tongji University School of Medicine, 389 Xincun Road, Shanghai 200065, P.R. China

E-mail: fanyuezu_shtj@yahoo.com.cn

Key words: astrocyte elevated gene-1, gallbladder neoplasm, prognosis as $85-100 \%$ at 5 years for lesions limited to the muscle layer (3). Despite considerable diagnostic and therapeutic advances in the treatment of GBC in recent years, the clinical outcome of GBC patients remains unsatisfactory (4), the 5-year survival rate after surgery is about $0-10 \%$ (5). However, to date, the molecular mechanism of its development and progression remains poorly understood. Hence, there is still an urgent need for further identification of novel molecular markers to provide the clinician with useful information concerning patient prognosis and possible therapeutic options.

Astrocyte elevated gene-1 (AEG-1), also known as metadherin (MTDH) (6) and originally identified as a protein induced in primary human fetal astrocytes infected with human immunodeficiency virus (HIV)-1 or treated with HIV gp120 or tumor necrosis factor- $\alpha$ (TNF- $\alpha$ ), has been demonstrated to be associated with the initiation and progression of cancer, the abrogation of cancer cell apoptosis and the induction of metastasis (7-10). The human AEG-1 gene, a downstream target of Harvey-Ras (Ha-ras), is located at chromosome 8q22, partly due to the phenomenon that the $8 \mathrm{q} 22$ gain functions independently in poor prognosis in breast cancer $(6,11)$. Recent observations have indicated that elevated AEG-1 expression increases anchorage-independent growth and invasion of malignant glioma (12), prostate cancer (13), neuroblastoma (14), HeLa (15), hepatocellular carcinoma cells (HCC) (16), esophageal squamous cell carcinoma (ESCC) (17) and breast cancer (18). Furthermore, AEG-1 siRNA expression could decrease proliferation and invasion of tumor cells. Recent studies have demonstrated that AEG-1 could exhibit important functions through several different molecular cascades, such as phosphoinositide 3-kinase (PI3K)/AKT $(8,19)$, NF- $\kappa \mathrm{B}(20,21)$, extracellular signal-regulated kinase (ERK) (16), Wnt/ $\beta$-catenin (22) and activator protein 1 (AP-1) (13) signaling pathway. In addition, AEG-1 has been confirmed to play a pivotal role in the process of angiogenesis (23). Interestingly, it has been reported that up-regulation of AEG-1 could increase and that the down-regulation of AEG-1 could decrease breast cancer cell metastasis in the lung (6). Furthermore, several lines of evidence revealed that elevated AEG-1 expression was significantly correlated with poor survival outcome and could be an independent prognostic factor in several carcinomas $(11-14,17,18,24,25)$. On the other hand, a recent study has reported that combination of 
chemotherapy with AEG-1 inhibition may help increase the efficacy of 5-fluorouracil-based combinatorial chemotherapy (26). These findings clearly indicate that AEG-1 is not only a potentially crucial mediator of tumor malignancy, but also a central converging point of complex oncogenic signaling networks.

Two cell lines were successfully established by our groups in 2003; the GBC-SD cell line and the human primary GBC cell line SGC-996, isolated from the primary mastoid adenocarcinoma of the gallbladder obtained from a 61-year-old female patient in Tongji Hospital, with the doubling time of cell proliferation being $48 \mathrm{~h}$. It has been shown that SGC-996 cells behave in accordance with the general characteristics of cell lines in vivo and in vitro and they have been applied in several laboratory investigations $(27,28)$.

However, to the best of our knowledge no study exists, examining the AEG-1 expression status in human primary GBC samples and GBC cell lines (GBC-SD and SGC-996) or the relationship between its expression and clinicopathological parameters or the AEG-1 relation with the prognosis of GBC patients.

In the present study, AEG-1 expression was up-regulated in GBC specimens and highly invasive GBC cell lines (GBC-SD) by immunohistochemistry (IHC), Western blot and real-time reverse transcription-PCR (RT-PCR)analysis. In addition, elevated AEG-1 expression exhibited significant correlation between its expression and differentiation degree, Nevin stage, Ki-67 expression and liver infiltration. More importantly, AEG-1 was an independent factor for the overall survival (OS) rate of GBC patients. Furthermore, low AEG-1 expression was associated with a better 5-year survival rate compared to patients with high AEG-1 expression.

\section{Materials and methods}

Patients and samples. This study was approved by the Research Ethics Review Board of Tongji University (Shanghai, China). Forty-one carcinomas, 10 adenomas and chronic or acute cholecystitis specimens of the gallbladder were obtained from patients that underwent operation and were histopathologically confirmed at Shanghai Tongji Hospital of Tongji University between January 2000 and December 2005. All GBC patients who had undergone surgical treatment and systematic integrated follow-up were investigated, including 16 males and 25 females aged 31-85 (median, 63) years. To reduce effects directly related to surgery, patients who died within one month after surgical resection were not included. The diagnosis of these GBC samples was verified by two independent pathologists who were blinded to the patients' clinical status. No patients had a history of chemotherapy or radiotherapy before surgery. According to the WHO criteria and the Nevin stage system, detailed pathological and clinical data were collected by reviewing medical charts and pathological records for all of the samples, including age, gender, tumor location, tumor size, histological grade, Nevin stage, liver infiltration, venous invasion and lymph node metastasis. According to the patients' clinical record, routine visit record, telephone number and address, clinical outcome was followed from the date of surgery to the date of death or until the end of December 31, 2010. Cases lost during follow-up were regarded
Table I. Demographic and clinicopathological parameters of GBC patients included in this study.

$\begin{array}{lc}\begin{array}{l}\text { Demographic and clinico- } \\ \text { pathological parameters }\end{array} & \text { Patients with } \\ \text { GBC (\%) }\end{array}$

No. of patients

Gender, $\mathrm{n}(\%)$

Male

Female

Age (years)

Median

Range

Tumor size $(\mathrm{cm})$

$3.03 \pm 0.68$

Tumor location, $\mathrm{n}(\%)$

Bottom

$22(53.7)$

Corporis and others

$19(46.3)$

Pathological diagnosis, $\mathrm{n}(\%)$

Adenocarcinoma

$35(85.4)$

Others $^{\mathrm{a}}$

Differentiation degree, $\mathrm{n}(\%)$

G1 (high)

G2 (moderate)

$10(24.4)$

G3 (poor)

$27(65.9)$

Nevin stage, $\mathrm{n}(\%)$

S1-S2

S3-S5

Lymph node metastasis, $\mathrm{n}(\%)$

$(-)$

15 (36.6)

$(+)$

$26(63.4)$

Liver infiltration, $\mathrm{n}(\%)$

$(-)$

$27(65.9)$

$(+)$

Venous invasion, $\mathrm{n}(\%)$

$(-)$

(+)

Curability, n (\%)

R0,1

$24(58.5)$

$\mathrm{R} 2$

$17(41.5)$

${ }^{\mathrm{a}}$ Others, i.e., squamous carcinoma, mucinous adenocarcinoma and adenosquamous carcinoma; GBC, gallbladder carcinoma.

as censored data for the survival analysis. Informed consent was obtained from each patient. All 41 patients from whom GBC specimens were obtained had been followed up. The median follow-up period for all patients was 26 (range, 1.5-60) months. The overall 5-year survival rate was $14.6 \%(6 / 41)$. Clinical and histopathological data are summarized in Table I.

Another 4 fresh GBC and matched adjacent non-cancerous tissue (ANT) samples, which were $>2 \mathrm{~cm}$ from the GBC tissues, were frozen and stored in liquid nitrogen until further use. 
Cell culture. Two established human GBC cell lines used in this study were the GBC-SD (Shanghai Cell Biology Research Institute of Chinese Academy of Sciences, CAS, China) and the SGC-996 (a generous gift from Dr Yao-Qing Yang, Tumor Cell Biology Research Institute of Tongji University, China). These cells were maintained and propagated in Dulbecco's modified Eagle's medium (DMEM, Gibco Co., USA) supplemented with $10 \%$ fetal bovine serum (FBS, Hangzhou Sijiqing Bioproducts, China) and $0.1 \%$ gentamicin sulfate (Gemini Bioproducts, Calabasas, CA). Cells were maintained at a $\log$ phase at $37^{\circ} \mathrm{C}$ with $5 \%$ carbon dioxide.

Invasion assay in vitro. The 35-mm,6-well Transwell membranes (Coster Co., USA) were used to measure the in vitro invasiveness of the two tumor cells. Briefly, a polyester (PET) membrane with $8-\mu \mathrm{m}$ pores was uniformly coated with a defined basement membrane matrix consisting of $50 \mu \mathrm{l}$ of Matrigel mixture diluted with serum-free DMEM (2:1 volume ratio) overnight at $4^{\circ} \mathrm{C}$ and used as the intervening barrier to invasion. The upper wells of the chamber were respectively filled with $1 \mathrm{ml}$ serum-free DMEM containing 2x105/ml tumor cells (GBC-SD or SGC-996 cells, $n=3$ ) and the lower wells of the chamber were filled with $3 \mathrm{ml}$ serum-free DMEM containing $1 \mathrm{X} \mathrm{MITO}^{+}$(Collaborative Biomedical, Bedford, MA). After $24 \mathrm{~h}$ in a humidified incubator at $37^{\circ} \mathrm{C}$ with $5 \%$ carbon dioxide, cells that had invaded through the basement membrane were stained with H\&E, and counted by light microscopy. Invasiveness was calculated as the number of cells that had successfully invaded through the matrix-coated membrane to the lower wells. Quantification was performed by counting the number of cells in 5 independent microscopic fields at a x400 magnification. Experiments were performed in duplicate and repeated three times with consistent results.

Immunohistochemical staining. IHC was performed as previously described (25). Briefly, after deparaffinizing, endogenous peroxide activity was inactivated. All tissue sections $(4 \mu \mathrm{m})$ were washed in phosphate-buffered saline (PBS, $\mathrm{pH}$ 7.4), then pretreated with $0.01 \mathrm{M}$ citrate buffer $(\mathrm{pH} 6.0$ ) for $30 \mathrm{~min}$ at $100^{\circ} \mathrm{C}$ in a microwave oven. Subsequently, the slides were allowed to cool at room temperature and washed in PBS again. Slides were then incubated respectively with rabbit polyclonal anti-AEG-1 protein IgG (Proteintech Group, Inc., USA; dilution 1:400) and mouse monoclonal anti-Ki-67 (BD Biosciences, USA; dilution 1:200, only for GBC samples) at $4^{\circ} \mathrm{C}$ overnight. After being rinsed with PBS again, the sections were incubated respectively with the goat anti-rabbit Envision kit (GK400305, Genetech, USA) for AEG-1 staining or with the goat antimouse Envision kit (GK400105, Genetech) for Ki-67 staining for $30 \mathrm{~min}$ at $37^{\circ} \mathrm{C}$ followed by incubation with 3,3'-diaminobenzidine (DAB) chromogen for $5 \mathrm{~min}$ at room temperature and washing with distilled water. Finally all of these sections were counterstained with hematoxylin. Negative controls were established by replacing the primary antibody with PBS in all samples. Known immunoassay-positive breast carcinoma sections were used as positive controls.

Western blot analysis. Total protein, including 4 cases of frozen GBC and counterpart ANT samples and two established human GBC cell lines (GBC-SD and SGC-996), were dissolved in RIPA butter for 5 min. Subsequently, cell debris was removed by centrifugation at $12,000 \mathrm{x}$ g for $10 \mathrm{~min}$. Using the bicinchoninic acid protein assay kit, protein concentrations were quantified and $30 \mu \mathrm{g}$ of protein per sample was separated onto a denaturing SDS-polyacrylamide gel (PAGE), and transferred onto PVDF membranes. Subsequently, membranes were blocked with $5 \%$ non-fat milk for $1 \mathrm{~h}$ followed by incubation overnight at $4^{\circ} \mathrm{C}$ with gentle agitation with the primary antibody, anti-AEG-1 (dilution 1:400, Proteintech Group, Inc.), diluted in buffer. After washing with TBST, the membranes were incubated with the secondary antibody (horseradish peroxidase-conjugated anti-rabbit) for $1 \mathrm{~h}$ at room temperature. The experiment was repeated in triplicate. Reactive protein was detected using the ECL chemiluminescence system (Pierce, Rockford, USA). The amount of each protein sample was normalized to $\beta$-actin (Sigma).

RNA extraction and real-time reverse transcription $(R T)-P C R$. Total RNA from cultured cell and surgically obtained tumor tissues was extracted using the TRIzol reagent (Invitrogen) and isolated according to the manufacturer's instructions. The first strand cDNA was synthesized according to the manufacturer's instructions (Takara RT kit, Dalian, China). Quantitative determination of AEG-1 transcript concentrations was performed by real-time RT-PCR with GAPDH as an internal control. Primers for AEG-1 (sense 5'-GGCAATTGGGTAGACGAAGA-3'; antisense 5'-CCTGTTTTGGACGGGTTTTA-3') and GAPDH (sense 5'-GAGTCAACGGATTTGGTCGT-3'; antisense 5'-TT GATTTTGGAGGGATCTCG-3') were synthesized by Sangon (Shanghai, China) and were used to measure gene expression. Amplification reaction assays were set up in triplicate for each sample using the SYBR-Green system (Takara). The PCR conditions were as follows: $95^{\circ} \mathrm{C}$ for $10 \mathrm{sec}$ for initial denaturation and 40 cycles of denaturation at $95^{\circ} \mathrm{C}$ for $5 \mathrm{sec}$ followed by annealing at $55^{\circ} \mathrm{C}$ for $35 \mathrm{sec}$. Real-time PCR was then employed to determine the fold increase of AEG-1 mRNA in each of the primary GBC tissues relative to the paired ANT tissues taken from the same patient and in the two established human GBC cell lines (GBC-SD and SGC-996). Expression data were normalized to the geometric mean of the housekeeping gene GAPDH to control the variability in the expression levels and analyzed using the $2^{-\Delta \Delta \mathrm{CT}}$ method described in a previous report (29), where $C_{T}$ represents the threshold cycle for each transcript.

Evaluation of immunohistochemistry. Positive AEG-1 expression was mainly localized in the cytoplasm in the vast majority of cancer tissues. However, a small number of cancer cells exhibited additional AEG-1 expression in the nucleus. AEG-1 was scored with IHC using a semi-quantitative system as previously described (18). Staining for AEG-1 was assessed in a series of randomly selected 10 high-power fields, and they were believed to be representative of the average in tumors at x400 magnification. The tumor cell proportion was scored as follows: 0 (no positive tumor cells), 1 ( $<10 \%$ positive tumor cells), 2 (10-50\% positive tumor cells), 3 ( $>50 \%$ positive tumor cells). The staining intensity was scored using a semi-quantitative four-category grading system: 0 , no staining; 1 , weak staining (light yellow); 2, moderate staining (yellow brown); 3, strong staining (brown). The staining index (SI) was calculated using the following formula: (staining intensity score) $\mathrm{x}$ (the 

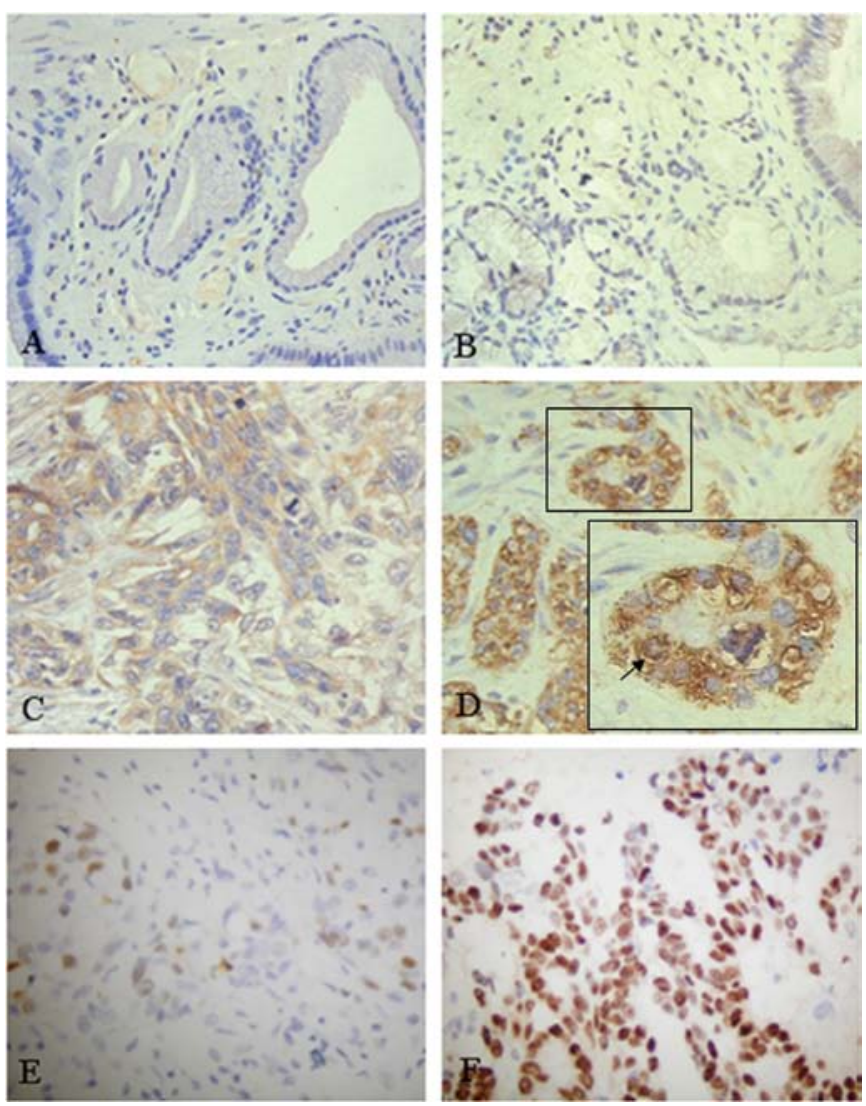

Figure 1. Immunohistochemical staining of AEG-1 and Ki-67 in different gallbladder tissues. (Envision kit, original magnification $\mathrm{x} 400$ ). (A) Chronic or acute cholecystitis mucosa; (B) Gallbladder adenoma; (C) Representative staining of low AEG-1 expression in gallbladder carcinoma (GBC) tissues (D) Representative staining of high AEG-1 expression in GBC tissues (arrows, nuclear staining). Representative staining of low (E) and high (F) $\mathrm{Ki}-67$ expression in GBC tissues.

proportion score of positive tumor cells). Using this method of assessment, we evaluated AEG-1 expression in GBC specimens by determining the SI, with scores of $0,1,2,3,4,6$ or 9 . The cut-off value for high and low expression level was chosen on the basis of a measure of heterogeneity with the log-rank test statistical analysis with respect to overall survival. The SI score of 4 (a cut-off point) was used to distinguish between low and high AEG-1 expression.

$\mathrm{Ki}$-67 staining mainly localized in the nuclear of proliferative cells. Only cells with a distinct nuclear Ki-67 staining were considered as positive, and the percentage of immunoreactive nuclei was calculated by counting in a series of randomly selected 10 microscopic fields (corresponding to a total of at least 100 tumor cells) under high-power magnification (x400). Finally, the patients were divided into two groups including $\mathrm{Ki}-67$-negative groups $(\leq 30 \%)$ and $\mathrm{Ki}-67$-positive groups $(>30 \%)(25)$.

Two experienced pathologists blinded to the clinical follow-up data independently scored the 41 GBC samples making up the cases used in this study. The complete score agreement of the two pathologists was $87.8 \%$ (36/41) of all cases, indicating that the scoring method was reasonably reproducible. A third blinded pathologist intervened and evaluated the patients with different IHC scores. If the third pathologist agreed with one of the previous scores, it was used for analysis.
Table II. Immunohistochemical analysis of AEG-1 expression in different gallbladder tissues.

\begin{tabular}{|c|c|c|c|c|}
\hline \multirow[b]{2}{*}{ Variable } & \multirow[b]{2}{*}{$\mathrm{n}$} & \multicolumn{2}{|c|}{$\begin{array}{c}\text { AEG-1 } \\
\text { expression }\end{array}$} & \multirow{2}{*}{$\frac{\chi^{2}}{\text { p-value }}$} \\
\hline & & Low & High & \\
\hline $\begin{array}{l}\text { Chronic or acute } \\
\text { cholecystitis }\end{array}$ & 10 & 10 & 0 & 0.0003 \\
\hline Gallbladder adenoma & 10 & 8 & 2 & \\
\hline Gallbladder carcinoma & 41 & 15 & 26 & \\
\hline
\end{tabular}

The three pathologists were asked to reach an agreement on the cases from which three different scores were obtained.

Statistical analysis. All data were analyzed using SAS 9.0 software (SAS Institute Inc., Cary, NC, USA). The $\chi^{2}$ test and the Fisher's exact test were used to analyze the relationship between AEG-1 expression and clinicopathological characteristics. Bivariate correlations between two independent variables were analyzed by calculating the Spearman's correlation coefficients. Association between categorical variables (expressed as mean \pm SD) was analyzed by the two-tailed Student's t-test. Survival curves were calculated with the Kaplan-Meier method and were compared using the log-rank test. Multivariate analysis of prognostic factors was performed using the Cox's regression model. A p-value $<0.05$ was considered statistically significant.

\section{Results}

Elevated AEG-1 expression in archived GBC tissue samples. In order to determine whether AEG-1 overexpression is correlated with different gallbladder tissues, from chronic or acute cholecystitis to gallbladder adenomas or carcinomas we performed IHC staining of paraffin-embedded, archived gallbladder tissues. Only weak or negative expression of AEG-1 expression was observed in all normal gallbladder mucosa specimens (Fig. 1A and B). The frequency and intensity of AEG-1 expression were gradually elevated from normal mucous specimens to adenomas and was highest in GBCs (Fig. 1C and D). Elevated AEG-1 expression was found in $63.4 \%$ (26 of 41) archived GBC specimens. It was noteworthy that no nuclear AEG-1 expression was observed in adenomas and normal tissues. Nuclear staining was observed only when AEG-1 was overexpressed, whereas low AEG-1 expression failed to exhibit a similar phenomenon. Furthermore, there were significant differences of AEG-1 expression between normal specimens, adenomas and GBCs (Table II, $\mathrm{p}=0.0003$ ). In addition, the AEG-1 expression showed no significant difference between chronic or acute cholecystitis and gallbladder adenoma tissues $(\mathrm{p}=0.4737)$. On the other hand, negative and positive expression of Ki-67 was observed in 13 and 28 of 41 GBC patients, respectively (Fig. 1E and F).

Invasive potential of GBC-SD and SGC-996 cells in vitro. The Transwell plates were used to measure the in vitro ability of cells to invade a basement membrane matrix, an important 
A

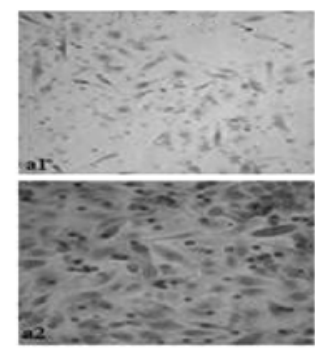

C

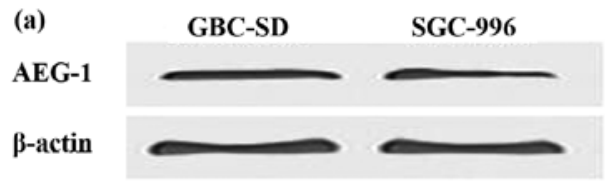

(b)

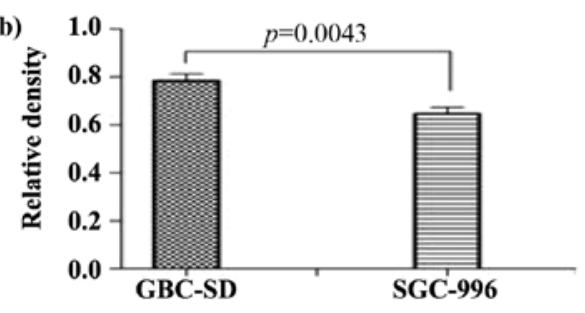

SGC-996

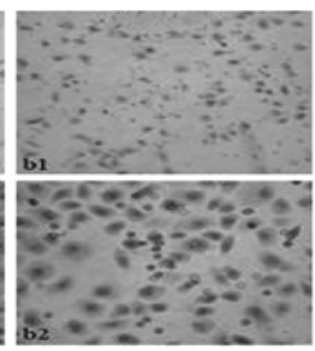

B

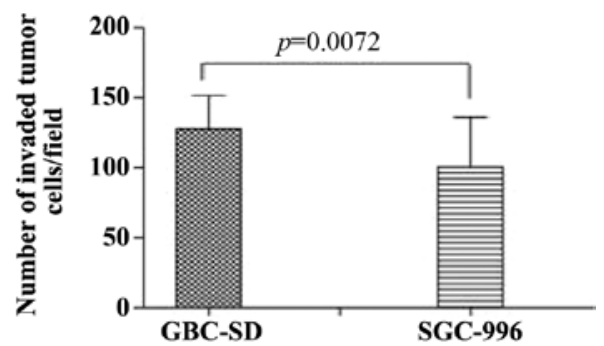

D
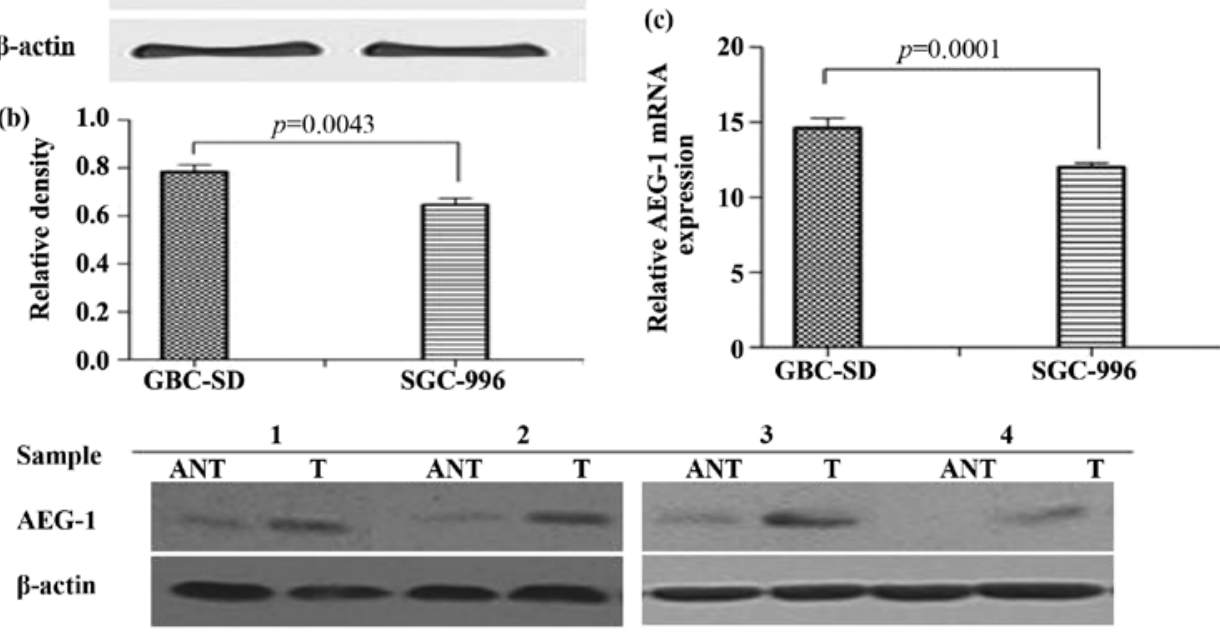

E
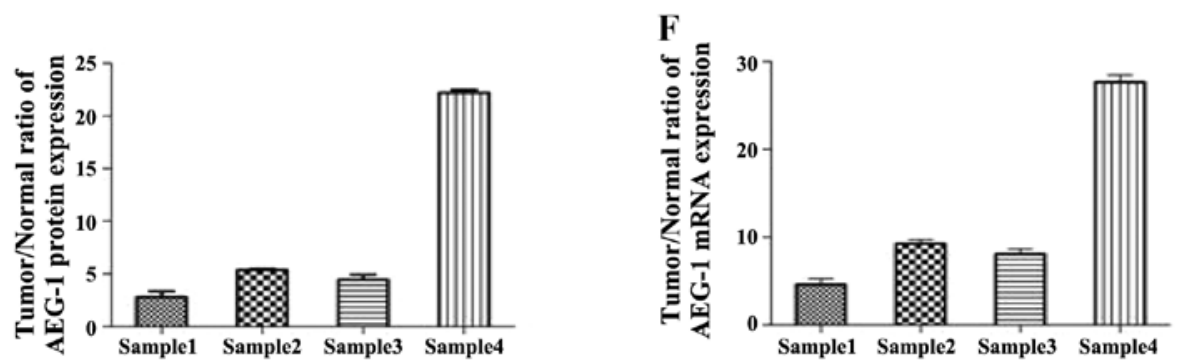

Figure 2. Invasive potential of GBC-SD and SGC-996 cell lines in vitro and elevated AEG-1 expression in gallbladder carcinoma (GBC). (A) Representative phase contrast microscopy pictures of GBC-SD cells (original magnification, a1, x200; a2 x400) and SGC-996 cells (original magnification, b1 x200; b2, x400) with H\&E staining. Both GBC-SD and SGC-996 cells could invade through the matrix-coated membrane to the lower wells of Transwell plates. (B) The invaded number of GBC-SD cells was higher than that of SGC-996 cells. Error bars represent standard deviations calculated from three parallel experiments. (C) Expression of AEG-1 protein in the cultured highly invasive GBC-SD cell lines and the relatively low invasive SGC-996 cell lines. Western blot analysis of AEG-1 expression in the GBC-SD and SGC-996 cell lines (a). Expression levels were normalized to $\beta$-actin. AEG-1 protein was more highly expressed in the GBC-SD cell line than in the SGC-996 cell line (b). Error bars represent standard deviations calculated from three parallel experiments. Real-time PCR analysis also showed that AEG-1 expression was significantly higher in GBC-SD cells than in SGC-996 cells (c). Expression levels were normalized to GAPDH. Expression (D) and quantification (E) of AEG-1 protein in each of the primary GBC (T) and adjacent non-cancerous tissue (ANT) paired from the same patient by Western blotting. Expression levels were normalized to $\beta$-actin. Error bars represent standard deviations. (F) Real-time PCR analysis of AEG-1 expression in the samples (1-4) of each of the primary GBC (T) and ANT paired from the same patient. Expression levels were normalized to GAPDH. Error bars represent standard deviations.

step in the metastatic cascade. We found the GBC-SD cells were mainly composed of spindle-shaped and polygonal cells. However, the SGC-996 cells could mainly form multi-layered colonies (Fig. 2A). Both GBC-SD and SGC-996 cells could successfully invade through the matrix-coated membrane to the lower wells. However, the number of GBC-SD cells was higher than that of SGC-996 cells $(127.7 \pm 23.86 \mathrm{vs}$ $100.5 \pm 27.07 ; \mathrm{t}=2.84, \mathrm{p}=0.0072$ ). Hence, the GBC-SD cell lines exhibited higher invasion potential than the relatively low invasive SGC-996 cell lines (Fig. 2A and B).
Increased expression of AEG-1 in fresh GBC tissue samples and cell lines. As shown in Fig. 2C, Western blot analysis showed that AEG-1 protein was more highly expressed in the GBC-SD cell line compared to the SGC-996 cell line ( $\mathrm{p}=0.0043$ ). Real-time RT-PCR was carried out to determine whether the difference in the AEG-1 expression levels was also apparent at the mRNA level. In agreement with the up-regulation of the AEG-1 protein levels in the GBC-SD cell line, these cells also showed significantly higher levels of AEG-1 mRNA compared to the SGC-996 cell line ( $\mathrm{p}=0.0001)$. 
Table III. Relationship between AEG-1 expression and clinicopathological data in gallbladder carcinoma.

\begin{tabular}{|c|c|c|c|c|c|}
\hline \multirow[b]{2}{*}{ Variables } & \multirow[b]{2}{*}{$\mathrm{n}$} & \multicolumn{2}{|c|}{ AEG-1 expression } & \multirow{2}{*}{$\frac{\chi^{2}}{\text { p-value }}$} & \multirow{2}{*}{$\begin{array}{c}\begin{array}{c}\text { Spearman rank } \\
\text { correlation }\end{array} \\
\mathrm{r}(\mathrm{p} \text {-value })\end{array}$} \\
\hline & & Low & High & & \\
\hline \multicolumn{6}{|l|}{ Gender } \\
\hline Male & 16 & 6 & 10 & 0.9225 & $0.0152(0.9249)$ \\
\hline Female & 25 & 9 & 16 & & \\
\hline \multicolumn{6}{|l|}{ Age (years) } \\
\hline$\leq 63$ & 18 & 9 & 9 & 0.1147 & $0.2404(0.1205)$ \\
\hline$>63$ & 23 & 6 & 17 & & \\
\hline \multicolumn{6}{|l|}{ Tumor size (cm) } \\
\hline$\leq 2$ & 17 & 5 & 12 & 0.4222 & $0.1254(0.4349)$ \\
\hline$>2$ & 24 & 10 & 14 & & \\
\hline \multicolumn{6}{|l|}{ Tumor location } \\
\hline Bottom & 22 & 7 & 15 & 0.4953 & $0.1065(0.5075)$ \\
\hline Corporis and others & 19 & 8 & 11 & & \\
\hline \multicolumn{6}{|l|}{ Pathological diagnosis } \\
\hline Adenocarcinoma & 35 & 14 & 21 & 0.2483 & $0.1712(0.2845)$ \\
\hline Others $^{\mathrm{a}}$ & 6 & 1 & 5 & & \\
\hline \multicolumn{6}{|l|}{ Differentiation degree } \\
\hline G1 (high) & 4 & 3 & 1 & $0.0259^{\mathrm{b}}$ & $0.4222(0.006)^{\mathrm{b}}$ \\
\hline G2 (moderate) & 10 & 6 & 4 & & \\
\hline G3 (poor) & 27 & 6 & 21 & & \\
\hline \multicolumn{6}{|l|}{ Nevin stage } \\
\hline $\mathrm{S} 1-\mathrm{S} 2$ & 9 & 6 & 3 & $0.0339^{\mathrm{b}}$ & $0.3312(0.0344)^{\mathrm{b}}$ \\
\hline S3-S5 & 32 & 9 & 23 & & \\
\hline \multicolumn{6}{|l|}{ Lymph node metastasis } \\
\hline$(-)$ & 15 & 7 & 8 & 0.3087 & $0.1589(0.3208)$ \\
\hline$(+)$ & 26 & 8 & 18 & & \\
\hline \multicolumn{6}{|l|}{ Liver infiltration } \\
\hline$(-)$ & 27 & 13 & 14 & $0.0328^{\mathrm{b}}$ & $0.3334(0.0332)^{\mathrm{b}}$ \\
\hline$(+)$ & 14 & 2 & 12 & & \\
\hline \multicolumn{6}{|l|}{ Venous invasion } \\
\hline$(-)$ & 28 & 12 & 16 & 0.3815 & $0.1911(0.2314)$ \\
\hline$(+)$ & 13 & 3 & 10 & & \\
\hline \multicolumn{6}{|l|}{ Ki-67 expression } \\
\hline$(-)$ & 13 & 9 & 4 & $0.0032^{\mathrm{b}}$ & $0.4618(0.0024)^{\mathrm{b}}$ \\
\hline$(+)$ & 28 & 6 & 22 & & \\
\hline
\end{tabular}

${ }^{\mathrm{a} O t h e r s, ~ i . e ., ~ s q u a m o u s ~ c a r c i n o m a, ~ m u c i n o u s ~ a d e n o c a r c i n o m a ~ a n d ~ a d e n o s q u a m o u s ~ c a r c i n o m a ; ~}{ }^{\mathrm{b}} \mathrm{p}<0.05$, statistically significant; AEG-1, astrocyte elevated gene-1.

To verify whether the up-regulation of AEG-1 is clinically associated with GBC progression, comparative analysis of AEG-1 expression was conducted on four cases of paired primary GBC tissue and ANT. The expression of AEG-1 protein was also found to be up-regulated in all four human primary GBC tissues compared with their matched ANT. Protein quantification showed that all four tumors displayed a $>2.8$-fold increase of AEG-1 protein compared with tissues adjacent to the tumors (Fig. 2D and E), using Western blotting. Importantly, real-time PCR analysis revealed that the tumor/normal ratio of AEG-1 message expression could be as high as 27.7-fold in one of the four paired primary GBC tissues (Fig. 2F). It is of note that the level of AEG-1 protein in the GBC cells and clinical GBC tissues was correlated with the mRNA expression level, indicating that the up-regulation of AEG-1 in GBC may be primarily caused by transcriptional up-regulation. 
Table IV. Univariate and multivariate analysis of overall survival rate of GBC patients with Cox proportional hazards model.

\begin{tabular}{|c|c|c|c|c|c|c|}
\hline \multirow[b]{2}{*}{ Variable } & \multicolumn{3}{|c|}{ Univariate analysis } & \multicolumn{3}{|c|}{ Multivariate analysis } \\
\hline & HR & $95 \% \mathrm{CI}$ & p-value & HR & $95 \% \mathrm{CI}$ & p-value \\
\hline \multicolumn{7}{|l|}{ Gender } \\
\hline Male vs. female & 0.620 & $0.242-1.590$ & 0.3195 & & & \\
\hline \multicolumn{7}{|l|}{ Age (years) } \\
\hline$\leq 63$ vs. $>63$ & 0.602 & $0.213-1.702$ & 0.3386 & & & \\
\hline \multicolumn{7}{|l|}{ Tumor location } \\
\hline Bottom vs. others & 0.589 & $0.213-1.629$ & 0.3079 & & & \\
\hline \multicolumn{7}{|l|}{ Tumor size (cm) } \\
\hline$\leq 2$ vs. $>2$ & 1.307 & $0.509-3.356$ & 0.5774 & & & \\
\hline \multicolumn{7}{|l|}{ Pathological diagnosis } \\
\hline Adenocarcinoma vs. others ${ }^{\mathrm{a}}$ & 0.705 & $0.185-2.680$ & 0.6076 & & & \\
\hline \multicolumn{7}{|l|}{ Histological differentiation } \\
\hline G1 vs. G2 and G3 & 1.736 & $0.612-4.930$ & 0.2999 & & & \\
\hline \multicolumn{7}{|l|}{ Nevin stage } \\
\hline S1,S2 vs. S3-S5 & 8.521 & $1.184-61.332$ & $0.0334^{\mathrm{b}}$ & 8.445 & $1.502-47.495$ & $0.0155^{\mathrm{b}}$ \\
\hline \multicolumn{7}{|l|}{ Venous invasion } \\
\hline$(-)$ Vs. (+) & 3.051 & $0.701-13.286$ & 0.1373 & & & \\
\hline \multicolumn{7}{|l|}{ Lymph node metastasis } \\
\hline (-) vs. (+) & 1.428 & $0.373-5.468$ & 0.6028 & & & \\
\hline \multicolumn{7}{|l|}{ Liver infiltration } \\
\hline$(-)$ vs. (+) & 3.688 & $1.004-13.545$ & $0.0493^{\mathrm{b}}$ & 4.626 & $1.579-13.522$ & $0.0052^{\mathrm{b}}$ \\
\hline \multicolumn{7}{|l|}{ Curability } \\
\hline R0,1 vs. R2 & 17.869 & $3.151-101.335$ & $0.0011^{\mathrm{b}}$ & 23.675 & $6.037-92.847$ & $0.0001^{\mathrm{b}}$ \\
\hline \multicolumn{7}{|l|}{ Ki-67 expression } \\
\hline (-) vs. (+) & 0.704 & $0.217-2.283$ & 0.3117 & & & \\
\hline \multicolumn{7}{|l|}{ AEG-1 expression } \\
\hline Low vs. high & 3.148 & $1.003-9.875$ & $0.0492^{\mathrm{b}}$ & 3.046 & $1.195-7.761$ & $0.0196^{\mathrm{b}}$ \\
\hline
\end{tabular}

Association between AEG-1 expression and clinicopathological data in $G B C$. We next explored the relationships between the expression of AEG-1 protein and clinicopathological data of GBC. As shown in Table III, no significant correlations were observed between the AEG-1 expression and the clinicopathological variables of age, gender, tumor location, tumor size, venous invasion, lymph node metastasis and pathological type (all $\mathrm{p}>0.05$ ).

However, increased AEG-1 expression in GBC specimens significantly correlated with differentiation degree $(\mathrm{p}=0.0259)$, Nevin stage $(\mathrm{p}=0.0339)$ and liver infiltration $(\mathrm{p}=0.0328)$. Furthermore, AEG-1 expression was related to the Ki-67 proliferation index $(\mathrm{p}=0.0032)$.

To further confirm the correlation between AEG-1 expression and clinicopathological parameters, Spearman rank correlation analysis was applied. Spearman rank correlations of AEG-1 expression levels to the differentiation degree, Nevin stage, Ki-67 expression and liver invasion were 0.4222 $(\mathrm{p}=0.006), 0.3312(\mathrm{p}=0.0344), 0.4618(\mathrm{p}=0.0024)$ and 0.3334 $(\mathrm{p}=0.0332)$, respectively (Table III).

Univariate and multivariate survival analysis. Factors involved in overall survival (OS) rate of GBC patients were identified using the Cox proportional hazards model (Table IV). Univariate analysis showed that liver infiltration $(p=0.0493)$, curability $(\mathrm{p}=0.0011)$, Nevin stage $(\mathrm{p}=0.0334)$ and AEG-1 expression $(\mathrm{p}=0.0492)$ were found to be the significant prognostic indicators for the OS rate of GBC patients, and were thereby selected as the parameters to be included in the same Cox regression model. Further multivariate analysis confirmed that the Nevin stage [Hazard ratio (HR), 8.445, 95\% confidence interval (CI), 1.502-47.495; $\mathrm{p}=0.0155]$, curability (HR, 23.675, 95\% CI, 6.037-92.847; $\mathrm{p}=0.0001)$, liver infiltration ( $\mathrm{HR}, 4.626 ; 95 \% \mathrm{CI}$, 1.579-13.522; $\mathrm{p}=0.0052$ ) and AEG-1 expression (HR, 3.046; $95 \% \mathrm{CI}, 1.195-7.761 ; \mathrm{p}=0.0196)$ were the independent prognostic factors for the OS rate of GBC patients. 


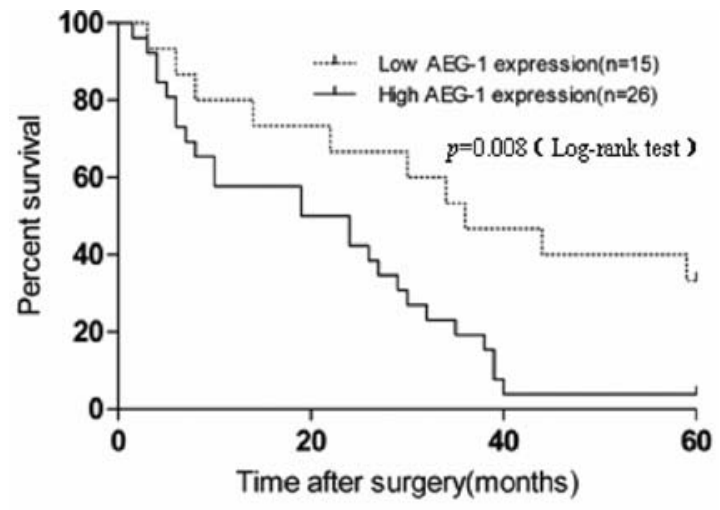

Figure 3. Kaplan-Meier survival curves for gallbladder carcinoma patients according to AEG-1 expression. The Kaplan-Meier plot of gallbladder carcinoma patients demonstrates a significantly low survival with high AEG-1 expression ( $\mathrm{p}=0.008, \log$-rank test).

Kaplan-Meier analysis and the log-rank test were used to further evaluate the AEG-1 expression on survival. The means and medians for survival time of the high AEG-1 expression group were 21.0 and 21.5 months, compared with 37.1 and 36 months for the low AEG-1 expression group. The cumulative 1-, 3- and 5-year OS rate were 57.7, 19.2 and 3.8\% in the high AEG-1 expression group and 80, 53.3 and $33.3 \%$ in the low AEG-1 expression group, respectively. The survival time of the high AEG-1 expression group was significantly shorter than that of the low AEG-1 expression group with a KaplanMeier analysis (Fig. 3, p=0.008, log-rank test).

\section{Discussion}

To our knowledge, this is the first report showing that AEG-1 was highly expressed in GBC samples and in the highly invasive GBC-SD cell line at both the protein and mRNA levels and strongly correlated with differentiation degree, Nevin stage, Ki-67 expression and liver infiltration in these patients. Furthermore, AEG-1, as an independent prognostic factor, was significantly associated with poor prognosis and reduced survival of GBC patients. Our results strongly suggest that AEG-1 protein may play a part in the modulation of GBC progression and could be a valuable biomarker for the prediction of GBC patient outcomes.

AEG-1 has been demonstrated to be up-regulated in several malignancies and to play a key role in Ha-ras-mediated oncogenesis through the PI3K/AKT/GSK3 $\beta / \mathrm{c}-$ Myc signaling pathway $(8,19)$. Several current studies have revealed that AEG-1 was a significant positive regulator of $N F-\kappa B$ and could enhance DNA binding and transcriptional activities of $\mathrm{NF}-\kappa \mathrm{B}$ in $\mathrm{HeLa}$ cells $(7,20)$. Furthermore, it is well known that NF- $\kappa B$ regulates the expression of various molecules which initiate cancer cell invasion and angiogenesis (20). It has been demonstrated that aberrant AEG-1 expression is a positive auto-feedback activator of AKT and a suppressor of FOXO3a in prostatic cancer cells (13). It has also been shown that AEG-1 could activate Wnt/ $\beta$-catenin signaling via ERK42/44 activation and could up-regulate lymphoid-enhancing factor $1 / \mathrm{T}$ cell factor 1 (LEF1/ TCF1) in human HCC (16). In addition, it has been reported that AEG-1 could augment matrix metalloproteinase-9 expres- sion and induce human glioma invasion (30). Taken together, depending on the cell types involved, AEG-1 could enhance various aspects of tumor malignancy including proliferation, invasion, metastasis and chemoresistance through different signaling pathways. Additionally, overexpression of AEG-1 has been correlated with carcinogenesis, progression and poor prognosis of various carcinomas, including breast cancer $(11,18)$, prostate cancer (13), malignant glioma (12), neuroblastoma (14), HCC (16), ESCC (17), colorectal cancer (24), endometrial cancer (25) and non-small cell lung cancer (NSCLC) (31). All these findings prompted us to ask whether AEG-1 is also clinically associated with the progression of GBC. To address this question, we explored the expression of AEG-1 in GBC cell lines and clinical paraffin-embedded, archived and fresh GBC tissue samples.

Our data showed that 26 out of the 41 (63.4\%) paraffinembedded, archived GBC tissues displayed overexpression of the AEG-1 protein by immunohistochemical analysis. On the other hand, only weak or negative expression of AEG-1 expression was observed in all normal gallbladder mucosa. Furthermore, real-time PCR analysis showed a 4.6- to 27.7-fold increase of the AEG-1 mRNA in primary GBC, as compared with the paired normal gallbladder tissues taken from the same patients, which is in line with previous studies in other types of carcinomas $(12-14,16-18,24,25)$, suggesting that AEG-1 may play a role in the development and progression of GBC. Moreover, we also found that the highly invasive GBC-SD cell line displayed elevated AEG-1 levels compared to the relatively low invasive SGC-996 cells, which is in agreement with observations from other groups. Several recent papers revealed that deregulation of AEG-1 could mediate migration and invasion of NSCLC (31) and hepatocellular carcinoma (16) and neuroblastoma (32) cell lines by AEG-1 siRNA technology. It has also been indicated that inhibition of $\mathrm{NF}-\kappa \mathrm{B}$ could block AEG-1-induced enhanced Matrigel invasion of HeLa cells (15). Although we failed to undertake AEG-1 siRNA technology in the current study, it is still reasonable to speculate that AEG-1 may also play an important role in the invasion potential of GBC patients. Further analysis of the relationship between AEG-1 expression and clinical characteristics of patients showed a significant correlation of its expression with differentiation degree, Nevin stage, Ki-67 expression (a proliferation marker), lymph node and liver metastasis. In addition, using multivariate analyses, we demonstrated that AEG-1 protein was an independent prognostic factor for the poor OS rate of GBC patients, which is consistent with previous findings $(12,13,16-18,24,25,31,32)$, strongly suggesting that AEG-1 may be useful as an independent marker to identify a subset of GBC patients with more aggressive disease and may be a predictor for the patient's prognosis and survival.

Interestingly, we also found that the nuclear staining of AEG-1 was present in advanced, metastatic GBC samples with high AEG-1 expression. However, a similar phenomenon was not found in the early stage GBC samples with low AEG-1 expression. These findings coincide with previous studies that AEG-1 resulted in the localization of the protein both in the cytoplasm and in the nucleus $(7,15,20,33)$. Under basal condition, AEG-1 as a novel transmembrane protein predominantly localizes in the perinuclear region and endoplasmic reticulum (15). However, upon forced overexpression, AEG-1 translocates 
into the nucleus, where it interacts with both the p65 subunit of $\mathrm{NF}-\kappa \mathrm{B}$ and the cyclic AMP-responsive element binding protein (CREB)-binding protein (CBP) $(7,20)$.

The most commonly used single agent in biliary tree tumors (including GBC) is 5-fluorouracil (5-FU). In general, 5 -FU has produced few responses, with response rates of $10-20 \%$ (34). However, several recent trials have suggested that 5 -FU in combination with other agents may produce better response rates (35-38). In light of the results published previously, AEG-1 could augment the expression of the multidrug resistance gene 1 (MDR1) protein, resulting in increased efflux and decreased accumulation of doxorubicin, promoting doxorubicin resistance in HCC (39). Another study showed that combination of chemotherapy with AEG-1 inhibition may help increase the efficacy of 5-FU-based combinatorial chemotherapy in HCC (26). These observations are provocative and raise the possibility of targeting AEG-1 as a novel idea for therapy of GBC.

In conclusion, the present study showed the AEG-1 expression was up-regulated in GBC specimens as well as in the highly invasive GBC-SD cell line, highlighting the clinical significance of AEG-1 in GBC. AEG-1 may serve as a valuable prognostic marker for GBC patients. However, further studies are need to clarify the mechanism by which AEG-1 is involved in the development and progression of GBC and to explore whether AEG-1 could be used as a novel chemotherapeutic target for $\mathrm{GBC}$ patients.

\section{Acknowledgements}

We are grateful to Professor An-Feng Fu and Professor Mei-Zheng Xi (Department of Pathology, Shanghai Jiaotong University, China) for their technical assistance with immunohistochemistry. We are also grateful to Profesor Chao $\mathrm{Lu}$ (Department of Pathophysiology, Fudan University, China) for his advice and technical assistance with Western blotting and real-time PCR analysis. In addition, we gratefully acknowledge access to the SGC-996 cell line provided by Professor Yao-Qing Yang (Tumor Cell Biology Research Institute, Medical College of Tongji University, China). In particular we thank Professor Han-Bao Tong (Department of Pathology, Shanghai Tenth People's Hospital, Tongji University, China) for reviewing the tissue specimens.

\section{References}

1. Miller G and Jarnagin WR: Gallbladder carcinoma. Eur J Surg Oncol 34: 306-312, 2008.

2. Hsing AW, Gao YT, Devesa SS, Jin F and Fraumeni JF Jr: Rising incidence of biliary tract cancers in Shanghai, China. Int J Cancer 75: 368-370, 1998.

3. Malka D, Boige V, Dromain C, Debaere T, Pocard M and Ducreux M: Biliary tract neoplasms: update 2003. Curr Opin Oncol 16: 364-371, 2004.

4. Zhu AX, Hong TS, Hezel AF and Kooby DA: Current management of gallbladder carcinoma. Oncologist 15: 168-181, 2010.

5. Misra S, Chaturvedi A, Misra NC and Sharma ID: Carcinoma of the gallbladder. Lancet Oncol 4: 167-176, 2003.

6. Brown DM and Ruoslahti E: Metadherin, a cell surface protein in breast tumors that mediates lung metastasis. Cancer Cell 5: 365-374, 2004

7. Emdad L, Sarkar D, Su ZZ, et al: Astrocyte elevated gene-1: recent insights into a novel gene involved in tumor progression, metastasis and neurodegeneration. Pharmacol Ther 114: 155-170, 2007.
8. Lee SG, Su ZZ, Emdad L, Sarkar D and Fisher PB: Astrocyte elevated gene-1 (AEG-1) is a target gene of oncogenic Ha-ras requiring phosphatidylinositol 3-kinase and c-Myc. Proc Natl Acad Sci USA 103: 17390-17395, 2006.

9. Kang DC, Su ZZ, Sarkar D, Emdad L, Volsky DJ and Fisher PB: Cloning and characterization of HIV-1-inducible astrocyte elevated gene-1, AEG-1. Gene 353: 8-15, 2005.

10. Su ZZ, Kang DC, Chen YM, et al: Identification and cloning of human astrocyte genes displaying elevated expression after infection with HIV-1 or exposure to HIV-1 envelope glycoprotein by rapid subtraction hybridization, RaSH. Oncogene 21: 3592-3602, 2002.

11. Hu G, Chong R, Yang Q, et al: MTDH Activation by $8 \mathrm{q} 22$ genomic gain promotes chemoresistance and metastasis of poorprognosis breast cancer. Cancer Cell 15: 9-20, 2009.

12. Emdad L, Sarkar D, Lee SG, et al: Astrocyte elevated gene-1: a novel target for human glioma therapy. Mol Cancer Ther 9: $79-88,2010$.

13. Kikuno N, Shiina H, Urakami S, et al: Knockdown of astrocyte elevated gene-1 inhibits prostate cancer progression through up-regulation of FOXO3a activity. Oncogene 26: 7647-7655, 2007.

14. Liu H, Song X, Liu C, Xie L, Wei L and Sun R: Knockdown of astrocyte elevated gene-1 inhibits proliferation and enhancing chemo-sensitivity to cisplatin or doxorubicin in neuroblastoma cells. J Exp Clin Cancer Res 28: 1-9, 2009.

15. Emdad L, Sarkar D, Su ZZ, et al: Activation of the nuclear factor kappa B pathway by astrocyte elevated gene-1: implications for tumor progression and metastasis. Cancer Res 66: 1509-1516, 2006.

16. Yoo BK, Emdad L, Su Z, et al: Astrocyte elevated gene-1 regulates hepatocellular carcinoma development and progression. J Clin Invest 119: 465-477, 2009.

17. Yu CP, Chen $\mathrm{K}$, Zheng HQ, et al: Overexpression of astrocyte elevated gene-1 (AEG-1) is associated with esophageal squamous cell carcinoma (ESCC) progression and pathogenesis. Carcinogenesis 30: 894-901, 2009.

18. Li J, Zhang N, Song LB, et al: Astrocyte elevated gene-1 is a novel prognostic marker for breast cancer progression and overall patient survival. Clin Cancer Res 14: 3319-3326, 2008.

19. Lee SG, Su ZZ, Emdad L, Sarkar D, Franke TF and Fisher PB: Astrocyte elevated gene-1 activates cell survival pathways through PI3K-Akt signaling. Oncogene 27: 1114-1121, 2008.

20. Sarkar D, Park ES, Emdad L, Lee SG, Su ZZ and Fisher PB: Molecular basis of nuclear factor-kappa $B$ activation by astrocyte elevated gene-1. Cancer Res 68: 1478-1484, 2008.

21. Khuda IIE, Koide N, Noman ASM, et al: Astrocyte elevated gene-1 (AEG-1) is induced by lipopolysaccharide as toll-like receptor 4 (TLR4) ligand and regulates TLR4 signalling. Immunology 128: e700-e706, 2009.

22. Hu GH, Wei Y and Kang YB: The multifaceted role of MTDH/ AEG-1 in cancer progression. Clin Cancer Res 15: 5615-5620, 2009.

23. Emdad L, Lee SG, Su ZZ, et al: Astrocyte elevated gene-1 (AEG-1) functions as an oncogene and regulates angiogenesis. Proc Natl Acad Sci USA 106: 21300-21305, 2009.

24. Song H, Li C, Li R and Geng J: Prognostic significance of AEG-1 expression in colorectal carcinoma. Int $\mathbf{J}$ Colorectal Dis 25: 1201-1209, 2010

25. Song H, Li C, Lu B, Zhang A and Geng S: Expression of astrocyte elevated gene-1: a novel marker of the pathogenesis, progression, and poor prognosis for endometrial cancer. Int J Gynecol Cancer 20: 1188-1196, 2010.

26. Yoo BK, Gredler R, Vozhilla N, et al: Identification of genes conferring resistance to 5-fluorouracil. Proc Natl Acad Sci USA 106: 12938-12943, 2009.

27. Chen Y, Jiang L, She F, et al: Vascular endothelial growth factor-C promotes the growth and invasion of gallbladder cancer via an autocrine mechanism. Mol Cell Biochem 345: 77-89, 2010.

28. Zong H, Yin B, Chen J, Ma B, Cai D and He X: Over-expression of c-FLIP confers the resistance to TRAIL-induced apoptosis on gallbladder carcinoma. Tohoku J Exp Med 217: 203-208, 2009.

29. Livak KJ and Schmittgen TD: Analysis of relative gene expression data using real-time quantitative PCR and the 2(T)(-Delta Delta C) method. Methods 25: 402-408, 2001.

30. Liu L, Wu J, Ying Z, et al: Astrocyte elevated gene-1 up-regulates matrix metalloproteinase-9 and induces human glioma invasion. Cancer Res 70: 3750-3759, 2010.

31. Song L, Li W, Zhang H, et al: Over-expression of AEG-1 significantly associates with tumour aggressiveness and poor prognosis in human non-small cell lung cancer. J Pathol 219: 317-326, 2009. 
32. Lee SG, Jeon HY, Su ZZ, et al: Astrocyte elevated gene-1 contributes to the pathogenesis of neuroblastoma. Oncogene 28: 2476-2484, 2009.

33. Li J, Yang L, Song L, et al: Astrocyte elevated gene-1 is a proliferation promoter in breast cancer via suppressing transcriptional factor FOXO1. Oncogene 28: 3188-3196, 2009.

34. Falkson G, Macintyre JM and Moertel CG: Eastern Cooperative Oncology Group experience with chemotherapy for inoperable gallbladder and bile-duct cancer. Cancer 54: 965-969, 1984.

35. Ducreux M, Rougier P, Fandi A, et al: Effective treatment of advanced biliary tract carcinoma using 5 -fluorouracil continuous infusion with cisplatin. Ann Oncol 9: 653-656, 1998.

36. Alberts SR, Al-Khatib H, Mahoney MR, et al: Gemcitabine, 5-fluorouracil, and leucovorin in advanced biliary tract and gallbladder carcinoma: a North Central Cancer Treatment Group phase II trial. Cancer 103: 111-118, 2005.
37. Feisthammel J, Schoppmeyer K, Mossner J, Schulze M, Caca K and Wiedmann M: Irinotecan with 5-FU/FA in advanced biliary tract adenocarcinomas: a multicenter phase II trial. Am J Clin Oncol 30: 319-324, 2007

38. Wagner AD, Buechner-Steudel P, Moehler M, et al: Gemcitabine, oxaliplatin and 5-FU in advanced bile duct and gallbladder carcinoma: two parallel, multicentre phase-II trials. Br J Cancer 101: 1846-1852, 2009.

39. Yoo BK, Chen D, Su ZZ, et al: Molecular mechanism of chemoresistance by astrocyte elevated gene-1. Cancer Res 70: 3249-3258, 2010. 